\title{
Refining PCR-based serotyping for detection of vaccine-preventable Streptococcus pneumoniae
}

\author{
Amanda L.S. Lang ${ }^{1}$, Hayley D. Gillis ${ }^{1}$, May Elsherif ${ }^{1}$, Irene Martin ${ }^{2}$, Todd F. Hatchette ${ }^{1}$, Shelly A. McNeil ${ }^{1}$, Jason J. \\ LeBlanc*1 \\ ${ }^{1}$ Canadian Center for Vaccinology (CCfV), IWK Health Centre, and Nova Scotia Health Authority (NSHA), Dalhousie University, \\ Halifax, NS, Canada \\ ${ }^{2}$ Streptococcus and STI Unit, National Microbiology Laboratory, Public Health Agency of Canada, Winnipeg, MB, Canada
}

Received: September 25, 2016

DOI: $10.5430 /$ jer.v3n1p28
Accepted: October 11, $2016 \quad$ Online Published: October 19, 2016

URL: http://dx.doi.org/10.5430/jer.v3n1p28

\begin{abstract}
Conventional multiplex PCR (cmPCR) reactions have been developed to monitor the most predominant serotypes of Streptococcus pneumoniae causing invasive pneumococcal disease (IPD). Since cmPCR assigns serotypes based on differences in the capsule biosynthesis (cps) loci, DNA extracted from clinical specimens can be used directly to monitor changes in serotype distribution and assess the impact of pneumococcal vaccines. Given that cmPCR can require up to eight reactions to assign a serotype, testing is often conducted in sequential algorithms. Sequential cmPCR reactions; however, may not be the most cost effective strategy to determine whether a $S$. pneumoniae serotype is vaccine-preventable. This study used oligonucleotide permutations in a modified set of cmPCR reactions (termed cmPCRmod) to reduce the number of PCR reactions required to identify S. pneumoniae serotypes covered by the 7- and 13-valent pneumococcal conjugate vaccines (PCV7 and PCV13, respectively) and the 23-valent pneumococcal polysaccharide vaccine (PPV23). While oligonucleotide permutations have previously been reported for regional differences in serotype distribution, the impact on assay performance had not been assessed. This study demonstrated that equivalent analytical sensitivity and specificity was seen when comparing cmPCR and cmPCRmod, and 100\% concordance was seen when 308 clinical isolates of $S$. pneumoniae were evaluated. Compared to cmPCR, cmPCRmod reduced the number and reactions required to detect serotypes covered by PCV7, PCV13, and PPV23. This study demonstrated that conventional multiplex reactions can be reformulated for more efficient detection of vaccine-preventable serotypes, without compromising test performance characteristics. As such, cmPCRmod reactions could provide significant cost savings for large surveillance studies.
\end{abstract}

Key Words: Streptococcus pneumoniae, PCR, Serotyping, Vaccine, Multiplex

\section{INTRODUCTION}

Streptococcus pneumoniae (or pneumococcus) is a bacterium that normally colonizes the human naso- and oropharynx but can also cause a spectrum of pneumococcal disease including community acquired pneumonia (CAP) and invasive pneumococcal diseases (IPD) such as meningitis and bac- teremia. $^{[1-4]}$ Both CAP and IPD are major causes of morbidity and mortality, and pose a significant burden on our healthcare system. ${ }^{[3,5]}$ Pneumococcal diseases are responsible for approximately 1.6 million deaths worldwide each year, with incidence rates greatest in children, the elderly, or individuals with risk factors. ${ }^{[6]}$

\footnotetext{
*Correspondence: Jason J. LeBlanc; Email: jason.leblanc@cdha.nshealth.ca; Address: Division of Microbiology, Department of Pathology and Laboratory Medicine, Nova Scotia Health Authority (NSHA), Room 404B, MacKenzie Building, 5788 University Avenue, Halifax, Nova Scotia, B3H 1V8, Canada.
} 
Childhood immunization programs have played an impor- Adults (CAPITA), ${ }^{[1]}$ recommendations were made for use tant role in reducing the burden of pneumococcal disease. of PCV13 in adults aged $\geq 65$ years. ${ }^{[12-14]}$ Both PCV13 Prior to introduction of the 7-valent pneumococcal conjugate vaccine (PCV7), most infections caused by $S$. pneumoniae were attributed to serotypes covered by this vaccine. ${ }^{[7-9]}$ While diseases caused by PCV7-serotypes have declined over the years, other serotypes have become predominant. This led to the use of the 13-valent pneumococcal conjugate vaccine (PCV13) in childhood immunization programs. ${ }^{[10]}$ and the 23-valent pneumococcal polysaccharide vaccines (PPV23) are also recommended for individuals at risk for pneumococcal disease. ${ }^{[12,13]}$ With the changing epidemiology of pneumococcal disease worldwide, monitoring the serotype distribution of S. pneumoniae is crucial to assess the impact of pneumococcal vaccines and help make informed Following the Community Acquired Pneumonia Trial In

recommendations for their use.

Table 1. Serotypes detected by the traditional and modified cmPCR reactions

\begin{tabular}{|c|c|c|c|c|c|c|c|c|c|}
\hline \multicolumn{3}{|c|}{ Vaccine Coverage } & \multirow{2}{*}{ Reaction } & \multirow{2}{*}{\multicolumn{6}{|c|}{ Serotype (Expected size in bp) }} \\
\hline PCV7 & PCV13 & PPV23 & & & & & & & \\
\hline \multirow[t]{2}{*}{$6 B$} & $\begin{array}{l}\text { 3,6A, 6B, } \\
19 \mathrm{~A}\end{array}$ & 3, 6B, 19A, 22F & cmPCR 1 & $\begin{array}{l}\text { cpsA } \\
(160)\end{array}$ & $\begin{array}{l}\text { 6A/6B/6C/6D } \\
(250)\end{array}$ & $\begin{array}{l}3 \\
(371)\end{array}$ & $\begin{array}{l}19 \mathrm{~A} \\
(566)\end{array}$ & $\begin{array}{l}22 \mathrm{~F} / 22 \mathrm{~A} \\
(643)\end{array}$ & $\begin{array}{l}16 \mathrm{~F} \\
(988)\end{array}$ \\
\hline & $7 \mathrm{~F}$ & $7 F, 8,33 F$ & cmPCR 2 & $\begin{array}{l}\text { cpsA } \\
(160)\end{array}$ & $\begin{array}{l}8 \\
(201)\end{array}$ & $\begin{array}{l}\text { 33F/33A/37 } \\
(338)\end{array}$ & $\begin{array}{l}15 \mathrm{~A} / 15 \mathrm{~F} \\
(434)\end{array}$ & $\begin{array}{l}23 \mathrm{~A} \\
(722)\end{array}$ & $\begin{array}{l}7 \mathrm{~F} / 7 \mathrm{~A} \\
(826)\end{array}$ \\
\hline $19 \mathrm{~F}$ & $19 \mathrm{~F}$ & $11 \mathrm{~A}, 12 \mathrm{~F}, 19 \mathrm{~F}$ & cmPCR 3 & $\begin{array}{l}\text { cpsA } \\
(160)\end{array}$ & $\begin{array}{l}19 \mathrm{~F} \\
(304)\end{array}$ & $\begin{array}{l}\text { 12F/12A/12B } \\
/ 44 / 46(376)\end{array}$ & $\begin{array}{l}11 \mathrm{~A} / 11 \mathrm{D} \\
(463)\end{array}$ & $\begin{array}{l}38 / 25 \mathrm{~F} / 25 \mathrm{~A} \\
(574)\end{array}$ & $\begin{array}{l}35 \mathrm{~B} \\
(677)\end{array}$ \\
\hline $4,9 \mathrm{~V}, 18 \mathrm{C}$ & $4,9 \mathrm{~V}, 18 \mathrm{C}$ & $4,9 \mathrm{~V}, 18 \mathrm{C}$ & cmPCR 4 & $\begin{array}{l}24 \mathrm{~A} / 24 \mathrm{~B} / 24 \mathrm{~F} \\
\text { (99) }\end{array}$ & $\begin{array}{l}\text { cpsA } \\
(160)\end{array}$ & $\begin{array}{l}\text { 7C/7B/40 } \\
(260)\end{array}$ & $\begin{array}{l}4 \\
(430)\end{array}$ & $\begin{array}{l}\text { 18C/18F/18B } \\
/ 18 \mathrm{~A}(573)\end{array}$ & $\begin{array}{l}9 \mathrm{~V} / 9 \mathrm{~A} \\
(816)\end{array}$ \\
\hline \multirow[t]{5}{*}{$14,23 \mathrm{~F}$} & $1,14,23 \mathrm{~F}$ & $\begin{array}{l}\text { 1, 10A, 14, 15B, } \\
23 \mathrm{~F}\end{array}$ & cmPCR 5 & $\begin{array}{l}\text { cpsA } \\
(160)\end{array}$ & $\begin{array}{l}14 \\
(189)\end{array}$ & $\begin{array}{l}1 \\
(280)\end{array}$ & $\begin{array}{l}23 \mathrm{~F} \\
(384)\end{array}$ & $\begin{array}{l}15 B / 15 C \\
(496)\end{array}$ & $\begin{array}{l}10 \mathrm{~A} \\
(628)\end{array}$ \\
\hline & 5 & $5,17 \mathrm{~F}$ & cmPCR 6 & $\begin{array}{l}39 \\
(98)\end{array}$ & $\begin{array}{l}\text { cpsA } \\
(160)\end{array}$ & $\begin{array}{l}10 \mathrm{~F} / 10 \mathrm{C} / / 33 \mathrm{C} \\
(248)\end{array}$ & $\begin{array}{l}5 \\
(362)\end{array}$ & $\begin{array}{l}35 \mathrm{~F} / 47 \mathrm{~F} \\
(517)\end{array}$ & $\begin{array}{l}17 \mathrm{~F} \\
(693)\end{array}$ \\
\hline & & 9N & cmPCR 7 & $\begin{array}{l}\text { cpsA } \\
(160)\end{array}$ & $\begin{array}{l}\text { 23B } \\
(199)\end{array}$ & $\begin{array}{l}35 \mathrm{~A} / 35 \mathrm{C} / 42 \\
(280)\end{array}$ & $\begin{array}{l}34 \\
(408)\end{array}$ & $\begin{array}{l}9 \mathrm{~N} / 9 \mathrm{~L} \\
(516)\end{array}$ & $\begin{array}{l}31 \\
(701)\end{array}$ \\
\hline & & 2,20 & cmPCR 8 & $\begin{array}{l}\text { cpsA } \\
(160)\end{array}$ & $\begin{array}{l}21 \\
(192)\end{array}$ & $\begin{array}{l}2 \\
(290)\end{array}$ & $\begin{array}{l}20 \\
(514)\end{array}$ & $\begin{array}{l}13 \\
(655)\end{array}$ & \\
\hline & & & cmPCR 6CD* & $\begin{array}{l}\text { cpsA } \\
(160)\end{array}$ & $\begin{array}{l}\text { 6A/6B/6C/6D } \\
(250)\end{array}$ & $\begin{array}{l}6 C / 6 D \\
(727)\end{array}$ & & & \\
\hline $14,19 \mathrm{~F}$ & $\begin{array}{l}3,7 \mathrm{~F}, 14,19 \mathrm{~A}, \\
19 \mathrm{~F}\end{array}$ & $\begin{array}{l}3,7 \mathrm{~F}, 14,19 \mathrm{~A}, \\
19 \mathrm{~F}\end{array}$ & cmPCRmod A & $\begin{array}{l}\text { cpsA } \\
(160)\end{array}$ & $\begin{array}{l}14 \\
(189)\end{array}$ & $\begin{array}{l}19 \mathrm{~F} \\
(304)\end{array}$ & $\begin{array}{l}3 \\
(371)\end{array}$ & $\begin{array}{l}19 \mathrm{~A} \\
(566)\end{array}$ & $\begin{array}{l}\text { 7F/7A } \\
(826)\end{array}$ \\
\hline \multirow[t]{7}{*}{$\begin{array}{l}4,6 \mathrm{~B}, 9 \mathrm{~V} \\
18 \mathrm{C}, 23 \mathrm{~F}\end{array}$} & $\begin{array}{l}\text { 4, 6A, 6B, 9V, } \\
18 \mathrm{C}, 23 \mathrm{~F}\end{array}$ & $\begin{array}{l}4,6 \mathrm{~B}, 9 \mathrm{~V}, 18 \mathrm{C}, \\
23 \mathrm{~F}\end{array}$ & cmPCRmod B & $\begin{array}{l}\text { cpsA } \\
(160)\end{array}$ & $\begin{array}{l}\text { 6A/6B/6C/6D } \\
(250)\end{array}$ & $\begin{array}{l}23 \mathrm{~F} \\
(384)\end{array}$ & $\begin{array}{l}4 \\
(430)\end{array}$ & $\begin{array}{l}\text { 18C/18F/18B } \\
/ 18 \mathrm{~A}(573)\end{array}$ & $\begin{array}{l}9 \mathrm{~V} / 9 \mathrm{~A} \\
(816)\end{array}$ \\
\hline & 1,5 & $1,5,15 \mathrm{~B}, 22 \mathrm{~F}$ & cmPCRmod C & $\begin{array}{l}\text { cpsA } \\
(160)\end{array}$ & $\begin{array}{l}1 \\
(280)\end{array}$ & $\begin{array}{l}5 \\
(362)\end{array}$ & $\begin{array}{l}15 B / 15 C \\
(496)\end{array}$ & $\begin{array}{l}22 \mathrm{~F} / 22 \mathrm{~A} \\
(643)\end{array}$ & $\begin{array}{l}6 \mathrm{C} / 6 \mathrm{D} \\
* \\
(727)\end{array}$ \\
\hline & & $\begin{array}{l}\text { 8, 9N, 10A, 11A, } \\
33 \mathrm{~F}\end{array}$ & cmPCRmod D & $\begin{array}{l}\text { cpsA } \\
(160)\end{array}$ & $\begin{array}{l}8 \\
(201)\end{array}$ & $\begin{array}{l}33 \mathrm{~F} / 33 \mathrm{~A} / 37 \\
(338)\end{array}$ & $\begin{array}{l}\text { 11A/11D } \\
(463)\end{array}$ & $\begin{array}{l}\text { 9N/9L } \\
(516)\end{array}$ & $\begin{array}{l}10 \mathrm{~A} \\
(628)\end{array}$ \\
\hline & & $2,12 \mathrm{~F}, 17 \mathrm{~F}, 20$ & cmPCRmod E & $\begin{array}{l}\text { cpsA } \\
(160)\end{array}$ & $\begin{array}{l}23 B \\
(199)\end{array}$ & $\begin{array}{l}2 \\
(290)\end{array}$ & $\begin{array}{l}12 \mathrm{~F} / 12 \mathrm{~A} / \\
12 \mathrm{~B} / 44 / 46 \\
(376)\end{array}$ & $\begin{array}{l}20 \\
(514)\end{array}$ & $\begin{array}{l}17 \mathrm{~F} \\
(693)\end{array}$ \\
\hline & & & cmPCRmod F & $\begin{array}{l}\text { cpsA } \\
(160)\end{array}$ & $\begin{array}{l}\text { 7C/7B/40 } \\
(260)\end{array}$ & $\begin{array}{l}15 \mathrm{~A} / 15 \mathrm{~F} \\
(434)\end{array}$ & $\begin{array}{l}13 \\
(655)\end{array}$ & $\begin{array}{l}23 \mathrm{~A} \\
(722)\end{array}$ & $\begin{array}{l}16 \mathrm{~F} \\
(988)\end{array}$ \\
\hline & & & cmPCRmod G & $\begin{array}{l}24 \mathrm{~A} / 24 \mathrm{~B} / 24 \mathrm{~F} \\
(99)\end{array}$ & $\begin{array}{l}\text { cpsA } \\
(160)\end{array}$ & $\begin{array}{l}\text { 10F/10C/33C } \\
(248)\end{array}$ & $\begin{array}{l}34 \\
(408)\end{array}$ & $\begin{array}{l}38 / 25 \mathrm{~F} / 25 \mathrm{~A} \\
(574)\end{array}$ & $\begin{array}{l}31 \\
(701)\end{array}$ \\
\hline & & & cmPCRmod H & $\begin{array}{l}39 \\
(98)\end{array}$ & $\begin{array}{l}\text { cpsA } \\
(160)\end{array}$ & $\begin{array}{l}21 \\
(192)\end{array}$ & $\begin{array}{l}35 \mathrm{~A} / 35 \mathrm{C} / 42 \\
(280)\end{array}$ & $\begin{array}{l}35 \mathrm{~F} / 47 \mathrm{~F} \\
(517)\end{array}$ & $\begin{array}{l}35 \mathrm{~B} \\
(677)\end{array}$ \\
\hline
\end{tabular}

*When a positive reaction is obtained in cmPCR1 for serotype 6A/6B/6C/6D, and additional reaction is performed to distinguish 6A/6B for 6C/D using cmPCR 6CD. In the modified reactions, serotype $6 \mathrm{~A} / 6 \mathrm{~B} / 6 \mathrm{C} / 6 \mathrm{D}$ is found in $\mathrm{cmPCRmodB}$ and the $6 \mathrm{CD}$ reaction is incorporated into cmPCRmod C.

To date, over 90 different S. pneumoniae serotypes have been identified using traditional Quellung serotyping, a microscopic method that classifies pneumococci based on capsule-specific antisera ${ }^{[15]}$ More recently, molecular methods for serotype deduction like conventional multiplex PCRs (cmPCR) have been developed, and are widely used from
DNA extracted from $S$. pneumoniae isolates or clinical specimens. ${ }^{[16-28]}$ Since cmPCR have a limited number of serotypes in each PCR reaction, they have been designed to target the most prevalent serotypes causing IPD, often in sequential reactions. ${ }^{[21-26]}$ However, sequential PCR may not be the most cost effective strategy to identify $S$. pneumo- 
niae serotypes that are vaccine-preventable. This study used oligonucleotide permutations in a modified set of cmPCR reactions (termed cmPCRmod) to reduce the amount of testing required to identify $S$. pneumoniae serotypes covered by the PCV7, PCV13, or PPV23 vaccines. By redistributing the primer pairs for vaccine-preventable serotypes, the number of cmPCRmod reactions required to span the coverage of PCV7, PCV13, and PPV23 was 2, 3, and 5, compared to the traditional cmPCR which require 4,6 , and 8 reactions, respectively (see Table 1 ).

\section{MethodS}

\subsection{S. pneumoniae source and culture}

For the analytical specificity analysis, each PCR-based serotyping method was tested against a panel of precharacterized S. pneumoniae isolates and non-pneumococcal streptococci that were obtained from one of five sources: the American Type Culture Collection (ATCC), the Centers for Disease Control and Prevention Global Pneumococcal Strain Bank (http: //www.cdc.gov/streplab/global-pneum o-strain-bank . html), the National Microbiology Laboratory (NML) in Winnipeg MB, Canada, the Serious Outcomes Surveillance (SOS) Network of the Canadian Immunization Research Network (CIRN) in Halifax, NS, Canada, ${ }^{[29]}$ or the biorepository in the Division of Microbiology at Nova Scotia Health Authority (NSHA), Halifax, NS, Canada.

S. pneumoniae isolates for the clinical validation $(\mathrm{n}=308)$ were obtained from two different sources: 87 S. pneumoniae specimens were collected as part of a national surveillance program for CAP and IPD by the CIRN SOS Network between Dec. 01, 2010 and Dec. 31, 2012. The other $221 \mathrm{~S}$. pneumoniae isolates (206 blood and 15 fluids: 8 cerebral spinal, 3 vitreous, 2 peritoneal, and 1 synovial) were collected as standard practice in the Division of Microbiology at NSHA (Halifax, NS) between June 2009 and December 2013. S. pneumoniae were characterized by Quellung serotyping using commercial pool, group, type and factor antisera (SSI Diagnostica; Statens Serum Institute, Copenhagen, Denmark) at the Streptococcus and STI Unit at the NML (Winnipeg, MB). ${ }^{[15,30]}$ All isolates were stored in skim milk at $-80^{\circ} \mathrm{C}$.

All specimens were cultured according to standard laboratory techniques. Pneumococcal isolates were confirmed by optochin disc susceptibility (Oxoid, Basingstoke, Hampshire, UK) and tube bile solubility analyses. ${ }^{[30,31]}$ All streptococci were cultured at $35^{\circ} \mathrm{C}$ in $5 \% \mathrm{CO}_{2}$ on trypticase soy agar (TSA) with 5\% sheep blood (Becton Dickinson, Mississauga, $\mathrm{ON})$. Bacterial growth was harvested from overnight cultures and suspended in phosphate-buffered saline (PBS) to a McFarland value of approximately 1.0 prior to nucleic acid extraction. The limit of detection (LoD) or analytical sensitivity of cmPCR and cmPCRmod was performed using three independent 10-fold serial dilutions (in PBS) of each identifiable $S$. pneumoniae serotype prior to nucleic acid extraction. The template nucleic acids extracted from each $S$. pneumoniae serotypes dilution were tested in triplicate with the respective cmPCR and cmPCRmod reactions used to identify each serotypes. Dilutions yielding $100 \%$ reproducibility $(n=9)$ for cmPCR or cmPCRmod reactions were assigned a representation of $1 \times \mathrm{LoD}$, thus representing the analytical sensitivity.

\subsection{Nucleic acid extraction and PCR-based serotyping}

Nucleic acids and cmPCR reactions were performed under conditions previously described (Lang et al., 2015). Nucleic acids were isolated from $200 \mu \mathrm{l}$ bacterial suspension using a MagNA Pure Total Nucleic Acid Isolation kit (Roche, Laval, QC) on a MagNA Pure LC instrument, as recommended by the manufacturer. Elution volume was set at $100 \mu \mathrm{l}$, and $5 \mu \mathrm{l}$ served as template for all PCR reactions. Both cmPCR and cmPCRmod reactions were performed in $25 \mu \mathrm{l}$ volumes that consisted of $1 \times$ enzyme mix from the Multiplex PCR kit (Qiagen Inc, Toronto, ON) with primer combinations and concentrations listed in Tables 1 and 2, respectively. For cmPCRmod, different primer combinations were used but the concentration of each remained the same as cmPCR reactions (see Tables 1, 2). All cmPCR and cmPCRmod reactions contained primers cmCpsA-F and cmCpsA-R which target the capsule biosynthesis gene a ( $c p s A)$ that is used as an internal control (see Tables 1, 2). Amplification for cmPCR and cmPCRmod were performed in 96-well plates using a C1000 thermocycler (Biorad Laboratories, Mississauga, $\mathrm{ON}$ ) as follows: $95^{\circ} \mathrm{C}$ for $90 \mathrm{~s}, 35$ cycles of $95^{\circ} \mathrm{C}$ for $30 \mathrm{~s}, 54^{\circ} \mathrm{C}$ for $90 \mathrm{~s}$, and $72^{\circ} \mathrm{C}$ for $60 \mathrm{~s}$, followed by $72^{\circ} \mathrm{C}$ for $10 \mathrm{~min}$. Amplicons were resolved using $1.2 \%$ agarose gel electrophoresis with $10 \mu \mathrm{g} / \mathrm{ml}$ ethidium bromide staining and visualized using a GelDoc XR + with ImageLab software (version 5.1) (Biorad Laboratories). Expected amplicon sizes in base pairs (bp) for cmPCR and cmPCRmod are denoted in Table 1. Oligonucleotides were synthesized by Integrated DNA Technologies (Coralville, IA).

\subsection{Cost analysis}

The cost account for nucleic acid extraction and reagent and consumable costs for the number of cmPCR or cmPCRmod reactions required, when processed sequentially, to identify S. pneumoniae serotypes covered by PCV7, PCV13, and PPV23. The overall cost savings of cmPCRmod was expressed as a percentage of the cost of cmPCR. 
Table 2. Oligonucleotides used in this study

\begin{tabular}{|c|c|c|c|c|}
\hline Name & Primer Sequence (5' to 3') & Concentration (nM) & Serotypes detected & Reference \\
\hline cmCpsA-F & GCA GTA CAG CAG TTT GTT GGA CTG ACC & 100 & \multirow{2}{*}{ All but 25A, 25F, and 38} & \multirow{2}{*}{ [25] } \\
\hline cmCpsA-R & GAA TAT TTT CAT TAT CAG TCC CAG TC & 100 & & \\
\hline $\mathrm{cm} 1-\mathrm{F}$ & CTC TAT AGA ATG GAG TAT ATA AAC TAT GGT TA & 300 & \multirow{2}{*}{1} & \multirow{2}{*}{ [25] } \\
\hline cm1-R & CCA AAG AAA ATA CTA ACA TTA TCA CAA TAT TGG C & 300 & & \\
\hline $\mathrm{cm} 2-\mathrm{F}$ & TAT CCC AGT TCA ATA TTT CTC CAC TAC ACC & 300 & \multirow{2}{*}{2} & \multirow{2}{*}{ [24] } \\
\hline $\mathrm{cm} 2-\mathrm{R}$ & ACA CAA AAT ATA GGC AGA GAG AGA CTA CT & 300 & & \\
\hline $\mathrm{cm} 3-\mathrm{F}$ & ATG GTG TGA TTT CTC CTA GAT TGG AAA GTA G & 300 & \multirow{2}{*}{3} & \multirow{2}{*}{ [25] } \\
\hline $\mathrm{cm} 3-\mathrm{R}$ & CTT CTC CAA TTG CTT ACC AAG TGC AAT AAC G & 300 & & \\
\hline $\mathrm{cm} 4-\mathrm{F}$ & CTG TTA CTT GTT CTG GAC TCT CGA TAA TTG G & 300 & \multirow{2}{*}{4} & \multirow{2}{*}{ [25] } \\
\hline $\mathrm{cm} 4-\mathrm{R}$ & GCC CAC TCC TGT TAA AAT CCT ACC CGC ATT G & 300 & & \\
\hline cm5-F & ATA CCT ACA CAA CTT CTG ATT ATG CCT TTG TG & 300 & \multirow{2}{*}{5} & \multirow{2}{*}{ [25] } \\
\hline $\mathrm{cm5} 5-\mathrm{R}$ & GCT CGA TAA ACA TAA TCA ATA TTT GAA AAA GTA TG & 300 & & \\
\hline $\mathrm{cm} 6 \mathrm{~A} / 6 \mathrm{~B} / 6 \mathrm{C} / 6 \mathrm{D}-\mathrm{F}$ & AAT TTG TAT TTT ATT CAT GCC TAT ATC TGG & 300 & \multirow{2}{*}{$6 \mathrm{~A}, 6 \mathrm{~B}, 6 \mathrm{C}, 6 \mathrm{D}$} & \multirow{2}{*}{ [25] } \\
\hline $\mathrm{cm} 6 \mathrm{~A} / 6 \mathrm{~B} / 6 \mathrm{C} / 6 \mathrm{D}-\mathrm{R}$ & TTA GCG GAG ATA ATT TAA AAT GAT GAC TA & 300 & & \\
\hline cm6C/6D-F & CAT TTT AGT GAA GTT GGC GGT GGA GTT & 500 & \multirow{2}{*}{$6 \mathrm{C}, 6 \mathrm{D}$} & \multirow{2}{*}{ [32] } \\
\hline cm6C/6D-R & AGC TTC GAA GCC CAT ACT CTT CAA TTA & 500 & & \\
\hline cm7C/7B-F & CTA TCT CAG TCA TCT ATT GTT AAA GTT TAC GAC GGG A & 300 & \multirow{2}{*}{ 7B, 7C, 40} & \multirow{2}{*}{ [25] } \\
\hline cm7C/7B-R & GAA CAT AGA TGT TGA GAC ATC TTT TGT AAT TTC & 300 & & \\
\hline cm7F/7A-F & CCT ACG GGA GGA TAT AAA ATT ATT GAG & 400 & \multirow{2}{*}{$7 \mathrm{~A}, 7 \mathrm{~F}$} & [25] \\
\hline cm7F/7A-R & CAA ATA CAC CAC TAT AGG CTG TTG AGA CTA AC & 400 & & [25] \\
\hline cm8-F & GAA GAA ACG AAA CTG TCA GAG CAT TTA CAT & 200 & 8 & [24] \\
\hline cm8-R & CTA TAG ATA CTA GTA GAG CTG TTC TAG TCT & 200 & 0 & {$[24]$} \\
\hline cm9N/9L-F & GAA CTG AAT AAG TCA GAT TTA ATC AGC & 500 & & \\
\hline cm9N/9L-R & ACC AAG ATC TGA CGG GCT AAT CAA T & 500 & 9L, 9N & [21] \\
\hline cm9V/9A-F & GGG TTC AAA G TC AGA CAG TG A ATC TTA A & 500 & & \\
\hline cm9V/9A-R & CCA TGA ATG A AA TCA ACA TT G TCA GTA GC & 500 & $9 \mathrm{~A}, 9 \mathrm{~V}$ & [24] \\
\hline cm10A-F & GGT GTA GAT TTA CCA TTA GTG TCG GCA GAC & 500 & & \\
\hline cm10A-R & GAA TTT CTT CTT TAA GAT TCG GAT ATT TCT C & 500 & $10 \mathrm{~A}$ & [25] \\
\hline $\mathrm{cm} 10 \mathrm{~F} / 10 \mathrm{C} / 33 \mathrm{C}-\mathrm{F}$ & GGA GTT TAT CGG TAG TGC TCA TTT TAG CA & 300 & $10 \mathrm{C}, 10 \mathrm{~F}, 33 \mathrm{C}$ & [24] \\
\hline $\mathrm{cm} 10 \mathrm{~F} / 10 \mathrm{C} / 33 \mathrm{C}-\mathrm{R}$ & CTA ACA AAT TCG CAA CAC GAG GCA ACA & 300 & (100, 105, & {$[24]$} \\
\hline cm11A/11D-F & GGA CAT GTT CAG GTG ATT TCC CAA TAT AGT G & 300 & 11A. 11D & [25] \\
\hline cm11A/11D-R & GAT TAT GAG TGT AAT TTA TTC CAA СТT СТC CC & 300 & $11 \mathrm{~A}, 1 \mathrm{NO}$ & {$[2 J]$} \\
\hline $\mathrm{cm} 12 \mathrm{~F} / 12 \mathrm{~A} / 44 / 46-\mathrm{F}$ & GCA ACA AAC GGC GTG AAA GTA GTT G & 500 & $12 \mathrm{~A} 12 \mathrm{~B}-12 \mathrm{~F}-44$ & [25] \\
\hline $\mathrm{cm} 12 \mathrm{~F} / 12 \mathrm{~A} / 44 / 46-\mathrm{R}$ & CAA GAT GAA TAT CAC TAC CAA TAA CAA AAC & 500 & $12 \mathrm{~A}, 12 \mathrm{D}, 12 \mathrm{~F}, 44,40$ & {$[25]$} \\
\hline cm13-F & TAC TAA GGT AAT CTC TGG AAA TCG AAA GG & 400 & 13 & [24] \\
\hline $\mathrm{cm} 13-\mathrm{R}$ & CTC ATG CAT TTT ATT AAC CGC TTT TTG TTC & 400 & 10 & {$[24]$} \\
\hline cm14-F & GAA ATG TTA CTT GGC GCA GGT GTC AGA ATT & 300 & 14 & [211] \\
\hline cm14-R & GCC AAT ACT TCT TAG TCT CTC AGA TGA AT & 300 & 14 & {$[21]$} \\
\hline $\mathrm{cm} 15 \mathrm{~A} / 15 \mathrm{~F}-\mathrm{F}$ & ATT AGT ACA GCT GCT GGA ATA TCT CTT C & 300 & $15 \mathrm{~A}, 15 \mathrm{~F}$ & [25] \\
\hline cm15A/15F-R & GAT CTA GTG AAC GTA CTA TTC CAA AC & 300 & $1 \mathrm{TA}, 1 \mathrm{TF}$ & [25] \\
\hline cm15B/15C-F & TTG GAA TTT TTT AAT TAG TGG CTT ACC TA & 300 & 15B, 15C & {$[25]$} \\
\hline cm15B/15C-R & CAT CCG CTT ATT AAT TGA AGT AAT CTG AAC C & 300 & (1) & \\
\hline cm16F-F & CTG TTC AGA TAG GCC ATT TAC AGC TTT AAA TC & 400 & $16 \mathrm{~F}$ & [25] \\
\hline cm16F-R & CAT TCC TTTTGTATA TAG TGC TAGTTC ATC C & 400 & $10 \mathrm{~F}$ & [25] \\
\hline cm17F-F & TTC GTG ATG ATA ATT CCA ATG ATC AAA CAA GAG & 500 & $17 \mathrm{~F}$ & [25] \\
\hline cm17F-R & GAT GTA ACA AAT TTG TAG CGA CTA AGG TCT GC & 500 & $1 / \mathrm{F}$ & \\
\hline $\mathrm{cm} 18 \mathrm{C} / 18 \mathrm{~F} / 18 \mathrm{~B} / 18 \mathrm{~A}-\mathrm{F}$ & CTT AAT AGC TCT CAT TAT TCT TTT TTT AAG CC & 300 & $18 \mathrm{~A}, 18 \mathrm{~B}, 18 \mathrm{C}, 18 \mathrm{~F}$ & {$[25]$} \\
\hline $\mathrm{cm} 18 \mathrm{C} / 18 \mathrm{~F} / 18 \mathrm{~B} / 18 \mathrm{~A}-\mathrm{R}$ & TTA TCT GTA AAC CAT ATC AGC ATC TGA AAC & 300 & 10R, 100, 100, 100 & {$[20]$} \\
\hline cm19A-F & GAG AGA TTC ATA ATC TTG CAC TTA GCC A & 300 & 194 & [33] \\
\hline cm19A-R & CAT AAT AGC TAC AAA TGA CTC ATC GCC & 300 & $13 \mathrm{~A}$ & [SS] \\
\hline cm19F-F & GTT AAG ATT GCT GAT CGA TTA ATT GAT ATC C & 500 & $19 F$ & [25] \\
\hline cm19F-R & GTA ATA TGT CTT TAG GGC GTT TAT GGC GAT AG & 500 & $13 \Gamma$ & {$[2 J]$} \\
\hline $\mathrm{cm} 20-\mathrm{F}$ & GAG CAA GAG TTT TTC ACC TGA CAG CGA GAA G & 300 & 20 & {$[25]$} \\
\hline $\mathrm{cm} 20-\mathrm{R}$ & CTA AAT TCC TGT AAT TTA GCT AAA ACT CTT ATC & 300 & 20 & {$[20]$} \\
\hline
\end{tabular}

(Table 2 continued on page 32) 
Table 2. (Continued.)

\begin{tabular}{|c|c|c|c|c|}
\hline Name & Primer Sequence (5’ to 3') & Concentration (nM) & Serotypes detected & Reference \\
\hline $\mathrm{cm} 21-\mathrm{F}$ & CTA TGG TTA TTT CAA CTC AAT CGT CAC C & 200 & \multirow{2}{*}{21} & \multirow{2}{*}[24]{} \\
\hline cm21-R & GGC AAA CTC AGA CAT AGT ATA GCA TAG & 200 & & \\
\hline $\mathrm{cm} 22 \mathrm{~F} / 22 \mathrm{~A}-\mathrm{F}$ & GAG TAT AGC CAG ATT ATG GCA GTT TTA TTG TC & 500 & \multirow{2}{*}{$22 \mathrm{~A}, 22 \mathrm{~F}$} & \multirow{2}{*}[25]{} \\
\hline $\mathrm{cm} 22 \mathrm{~F} / 22 \mathrm{~A}-\mathrm{R}$ & CTC CAG CAC TTG CGC TGG AAA CAA CAG ACA AC & 500 & & \\
\hline cm23A-F & TAT TCT AGC AAG TGA CGA AGA TGC G & 500 & \multirow{2}{*}{$23 \mathrm{~A}$} & \multirow{2}{*}[24]{} \\
\hline cm23A-R & CCA ACA TGC TTA AAA ACG CTG CTT TAC & 500 & & \\
\hline cm23B-R & GTC CAC GCT GAA TAA AAT GAA GCT CCG & 200 & 23B & {$[24]$} \\
\hline cm23F-F & GTA ACA GTT GCT GTA GAG GGA ATT GGC TTT TC & 500 & \multirow{2}{*}{$23 \mathrm{~F}$} & \multirow{2}{*}{ [25] } \\
\hline cm23F-R & CAC AAC ACC TAA CAC TCG ATG GCT ATA TGA TTC & 500 & & \\
\hline $\mathrm{cm} 24 \mathrm{~F} / 24 \mathrm{~A} / 24 \mathrm{~B}-\mathrm{F}$ & GCT CCC TGC TAT TGT AAT CTT TAA AGA G & 200 & \multirow{2}{*}{$24 \mathrm{~A}, 24 \mathrm{~B}, 24 \mathrm{~F}$} & \multirow{2}{*}[24]{} \\
\hline $\mathrm{cm} 24 \mathrm{~F} / 24 \mathrm{~A} / 24 \mathrm{~B}-\mathrm{R}$ & GTG TCT TTT ATT GAC TTT ATC ATA GGT CGG & 200 & & \\
\hline cm31-F & GGA AGT TTT CAA GGA TAT GAT AGT GGT GGT GC & 500 & 31 & {$[25]$} \\
\hline cm33F/33A/37-F & GAA GGC AAT CAA TGT GAT TGT GTC GCG & 300 & \multirow{2}{*}{ 33A, 33F, 37} & \multirow{2}{*}{ [25] } \\
\hline cm33F/33A/37-R & CTT CAA AAT GAA GAT TAT AGT ACC CTT CTA C & 300 & & \\
\hline $\mathrm{cm} 34-\mathrm{F}$ & GCT TTT GTA AGA GGA GAT TAT TTT CAC CCA AC & 300 & \multirow{2}{*}{34} & \multirow{2}{*}[25]{} \\
\hline cm34-R & CAA TCC GAC TAA GTC TTC AGT AAA AAA CTT TAC & 300 & & \\
\hline $\mathrm{cm} 35 \mathrm{~A} / 35 \mathrm{C} / 42-\mathrm{F}$ & ATT ACG ACT CCT TAT GTG ACG CGC ATA & 300 & \multirow{2}{*}{$35 \mathrm{~A}, 33 \mathrm{C}, 42$} & \multirow{2}{*}[24]{} \\
\hline $\mathrm{cm} 35 \mathrm{~A} / 35 \mathrm{C} / 42-\mathrm{R}$ & CCA ATC CCA AGA TAT ATG CAA CTA GGT T & 300 & & \\
\hline cm35B-F & GAT AAG TCT GTT GTG GAG ACT TAA AAA GAA TG & 500 & \multirow{2}{*}{ 35B } & \multirow{2}{*}[25]{} \\
\hline cm35B-R & CTT TCC AGA TAA TTA CAG GTA TTC CTG AAG CAA G & 500 & & \\
\hline $\mathrm{cm} 35 \mathrm{~F} / 47 \mathrm{~F}-\mathrm{F}$ & GAA CAT AGT CGC TAT TGT ATT TTA TTT AAA GCA A & 300 & \multirow{2}{*}{$35 F, 47 F$} & \multirow{2}{*}[25]{} \\
\hline $\mathrm{cm} 35 \mathrm{~F} / 47 \mathrm{~F}-\mathrm{R}$ & GAC TAG GAG CAT TAT TCC TAG AGC GAG TAA ACC & 300 & & \\
\hline $\mathrm{cm} 38 / 25 \mathrm{~F} / 25 \mathrm{~A}-\mathrm{F}$ & CGT TCT TTT ATC TCA CTG TAT AGT ATC TTT ATG & 300 & \multirow{2}{*}{$25 \mathrm{~A}, 25 \mathrm{~F}, 38$} & \multirow{2}{*}[25]{} \\
\hline cm38/25F/25A-R & ATG TTT GAA TTA AAG CTA ACG TAA CAA TCC & 300 & & \\
\hline cm39-F & TCA TTG TAT TAA CCC TAT GCT TTA TTG GTG & 200 & 39 & {$[24]$} \\
\hline
\end{tabular}

Table 3. Bacterial strains used in the specificity analysis

\begin{tabular}{|c|c|c|}
\hline \multirow{2}{*}{ Strains } & \multicolumn{2}{|l|}{ Results } \\
\hline & cmPCR & cmPCRmod \\
\hline S. pneumoniae ATCC 49619, serotype 19F & Pos. & Pos. \\
\hline S. pneumoniae (clinical isolates), serotype: & & \\
\hline $\begin{array}{l}\text { 1, 2, 3, 4, 5, 6A, 6B, 6C, 6D, 7A, 7F, 9A, 9V, 11A, 11D, 12A,12F, 14, 15A, 15F,16F, 18A, 18B, 18C, 18F, } \\
19 \mathrm{~A}, 19 \mathrm{~F}, 22 \mathrm{~A}, 22 \mathrm{~F}, 23 \mathrm{~A}, 23 \mathrm{~F}, 33 \mathrm{~A}, 33 \mathrm{~F}, 37,44 \text {, and 46 }\end{array}$ & Pos. & Pos. \\
\hline S. pneumoniae (clinical isolates), serotype: & & \\
\hline $\begin{array}{l}\text { 7B, 7C, 8, 9L, 9N, 10A, 10C, 10F, 12B, 13, 15B, 15C, 17F, 20, 21, 23B, 24A, 24B, 24F, 25A, 25F, 31, 33C, 34, } \\
35 \mathrm{~A}, 35 \mathrm{~B}, 35 \mathrm{C}, 35 \mathrm{~F}, 38,39,40,42 \text {, and 47F }\end{array}$ & Pos. & Pos. \\
\hline $\begin{array}{l}\text { S. pneumoniae (clinical isolates), serotype: 10B, 11B, 11C, 11F, 16A, 17A, 19B, 19C, 27, 28A, 28F, 29, 32F, } \\
33 \mathrm{~B}, 36,41 \mathrm{~A}, 41 \mathrm{~F}, 43,45,47 \mathrm{~A}, 48\end{array}$ & NT & NT \\
\hline S. agalactiae ATCC 12386 & Neg. & Neg. \\
\hline S. dysgalactiae subsp. equisimilis ATCC 12388 & Neg. & Neg. \\
\hline S. equi subsp. zooepidemicus ATCC 700400 and 43079 & Neg. & Neg. \\
\hline S. gallolyticus ATCC 9809 & Neg. & Neg. \\
\hline S. gordonii ATCC 33399 & Neg. & Neg. \\
\hline S. mitis ATCC 49456 & Neg. & Neg. \\
\hline S. mutans ATCC 25175 & Neg. & Neg. \\
\hline S. oralis ATCC 35037 & Neg. & Neg. \\
\hline S. pseudopneumoniae ATCC BAA-960 & Neg. & Neg. \\
\hline S. pyogenes ATCC 19615 & Neg. & Neg. \\
\hline S. salivarius subsp. thermophilus ATCC 19258 & Neg. & Neg. \\
\hline S. sanguinis ATCC 10556 & Neg. & Neg. \\
\hline S. uberis ATCC 700407 & Neg. & Neg. \\
\hline
\end{tabular}




\section{Results}

\subsection{Analytical specificity and sensitivity}

Each of the PCR-based serotyping assays (cmPCR and cmPCRmod) was highly specific, and no cross reactivity occurred between the various serotypes or with nonpneumococcal streptococci (see Table 3). As previously reported, confounding amplicons (as a result of non-specific amplification) sometimes occurred with cmPCR and cmPCRmod reactions. ${ }^{[19]}$ If present, these could readily be resolved with repeat reactions using individual primer pairs targeting the suspected serotype. Normalization of the S. pneumoniae culture to a McFarland value of 1.0 prior to extraction provided sufficient template that gave strong and reliable amplicons for all detectable serotypes. Based on end-point titers at $1 \times$ LoD for each detectable serotype, no differences in analytical sensitivity were seen between cmPCR and cmPCRmod.

As shown in Table 3, cmPCR and cmPCRmod reactions were equivalent in their abilities to detect $S$. pneumoniae serotypes, and did not cross-react with any other Streptococcus species. Abbreviations: conventional multiplex PCR (cmPCR), modified conventional multiplex PCR (cmPCRmod), and non-typeable (NT).

\subsection{Clinical isolate testing}

No discordant results were observed between cmPCR and cmPCRmod, and a serotype could be assigned for $99.7 \%$ (307/308) of S. pneumoniae isolates. A single non-typeable (NT) isolate was detected as cpsA-positive by the internal control, and was identified as serotype 28A by Quellung serotyping (see Figure 1). The overall trend in serotype distribution obtained by cmPCRmod mirrored Quellung serotyping, showing a predominance of serotypes 3, 7F, and 19A (see Figure 1). Quellung serotyping showed that $5 \%$ of S. pneumoniae were PCV7 serotypes, 54\% were PCV13 serotypes, and 74\% were PPV23 serotypes (see Figure 1A). Similarly, cmPCR and cmPCRmod demonstrated 7\%, 54\%, and $77 \%$ for serotypes covered by PCV7, PCV13, and PPV23, respectively (see Figure 1B). Focusing on select reactions containing vaccine-preventable serotypes, the number of $\mathrm{cmPCR}$ reactions required to span the coverage of PCV7, PCV13, and PPV23 was 4, 6, and 8, compared to 2, 3 , and 5 with cmPCRmod, respectively (see Table 1). When applied to the 308 S. pneumoniae isolates used in this study, cmPCRmod reduced the total number of reactions required to identify vaccine-preventable serotypes, leading to significant cost savings (see Table 4).

Published by Sciedu Press

\section{A) Quellung serotyping}

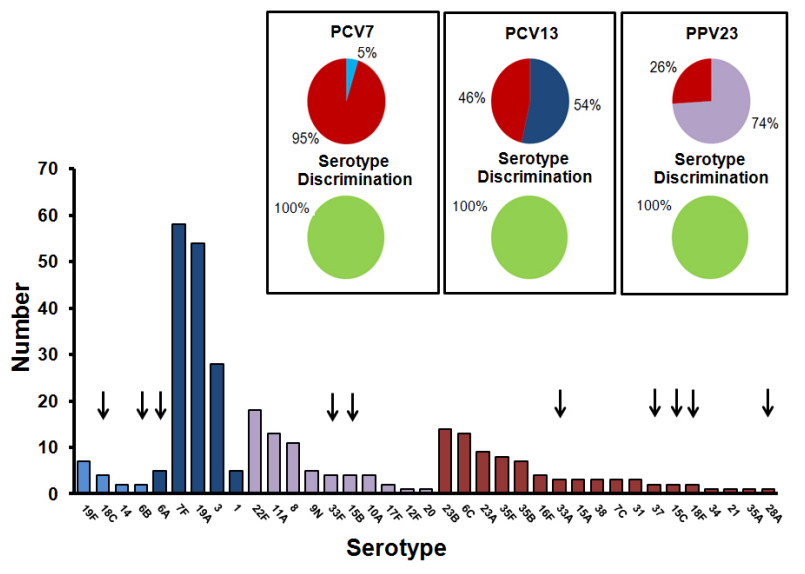

B) CmPCR or cmPCRmod

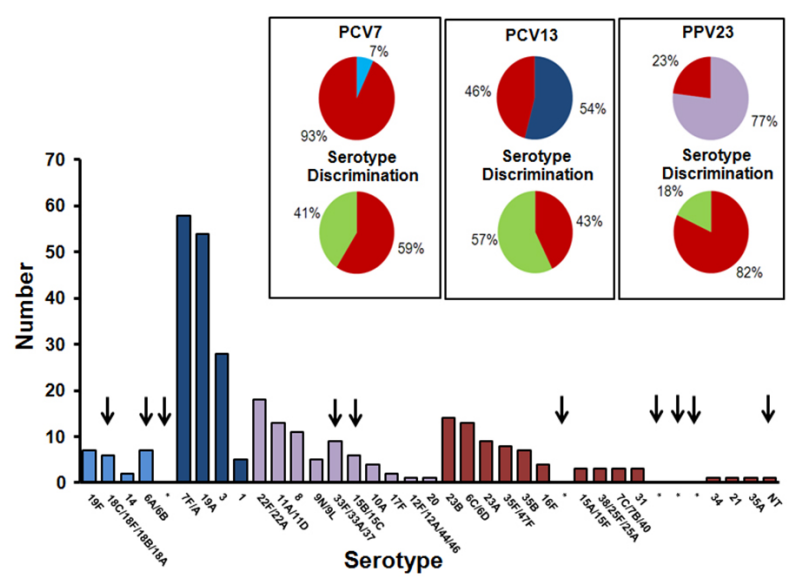

Figure 1. S. pneumoniae serotypes distribution using Quellung and PCR-based serotyping. Arrows highlight differences between results for: A) Quellung serotyping, and B) cmPCR or cmPCRmod. The pie charts on the top of the inset show the proportions of PCV7 (pale blue), PCV13 (dark blue), and PPV23 (magenta) serotypes. The same colors are used in the histogram, and non-vaccine serotypes are in red. The pie charts on the bottom of the inset show the proportion of serotypes that are fully differentiated (green) or lack discrimination (red)

Table 4. Number of PCR reactions required to identify vaccine-preventable serotypes of $S$. pneumoniae $(\mathrm{n}=308)$

\begin{tabular}{llll}
\hline \multirow{2}{*}{$\begin{array}{l}\text { Vaccine } \\
\text { coverage }\end{array}$} & \multicolumn{3}{l}{ Number of PCR reactions (cost in \$CAD) } \\
\cline { 2 - 4 } & cmPCR & cmPCRmod & Cost reduction (\%) \\
\hline PCV7 & $1232(5,904.36)$ & $616(4,338.18)$ & 26.5 \\
PCV13 & $1848(7,470.54)$ & $924(5,121.27)$ & 31.4 \\
PPV23 & $2464(9,036.72)$ & $1540(6,687.45)$ & 36.0 \\
\hline
\end{tabular}

As shown in Table 4, the number of reactions required to identify vaccine-preventable serotypes is lower in cmpCR- 
mod compared to cmPCR, leading to significant cost savings.

\section{Discussion}

Accurate identification of vaccine-preventable serotypes of $S$. pneumoniae can help determine the burden of disease caused by these serotypes, identify high-risk populations that could benefit from pneumococcal vaccination, and be used to evaluate vaccine effectiveness over time. In contrast to traditional testing algorithms based solely on serotype prevalence, this study used modified PCR reactions that focused on the identification of $S$. pneumoniae serotypes covered by PCV7, PCV13, and PPV23. When applied to 308 clinical isolates of S. pneumoniae, cmPCRmod reduced the total number of reactions required for the identification of vaccine-preventable serotypes.

It should be noted that molecular serotyping of $S$. pneumoniae based on differences in the capsular biosynthesis genes like cmPCR, real-time PCR, microarrays, hybridization assays, and sequencing, all suffer from the same limitation: failure to discriminate between some pneumococcal serotypes. ${ }^{[19,34-39]}$ For example, while serotype $7 \mathrm{~F}$ is covered in all three pneumococcal vaccines, $7 \mathrm{~F}$ cannot be discriminated from serotype 7A using these molecular methods, and therefore the serotype is assigned 7F/7A. Other vaccinepreventable serotypes identified by PCR-based serotyping cannot discriminate: $6 \mathrm{~A}$ from $6 \mathrm{~B}$; $9 \mathrm{~V}$ from $9 \mathrm{~A}$; $9 \mathrm{~N}$ from $9 \mathrm{~L}$; 11A from $11 \mathrm{D} ; 12 \mathrm{~F}$ from serotypes $12 \mathrm{~A}, 12 \mathrm{~B}, 44$, and 46 ; $15 \mathrm{~B}$ from $15 \mathrm{C} ; 18 \mathrm{C}$ from serotypes $18 \mathrm{~F}, 18 \mathrm{~B}$, and $18 \mathrm{~A} ; 22 \mathrm{~F}$ from $22 \mathrm{~A} ; 33 \mathrm{~F}$ from serotypes $33 \mathrm{~A}$ and 37 . For accurate discrimination of vaccine-preventable serotypes, these PCR groups would have to be resolved.

In this study, all vaccine-preventable serotypes identified by Quellung serotyping were also detected with cmPCRmod and cmPCR (see Figure 1). On the other hand, the proportion of serotypes covered by PCV7, PCV13, and PPV23 that could be fully discriminated was $41 \%, 57 \%$, and $18 \%$, respectively (see Figure 1B). The remaining proportions contained serotypes that were termed "possibly" vaccinepreventable. To overcome this limitation, some laboratories assign serotypes based on assumptions from serotype prevalence (where serotype 7F/7A identified by PCR would be considered $7 \mathrm{~F}$, the most prevalent serotype). Using all eight cmPCR and cmPCRmod reactions, the serotype distribution of pneumococcal isolates was identical and mirrored results obtained with Quellung serotyping, with only subtle differences (see Figure 1). A high level of accuracy for the detection of serotypes covered by PCV7 (98.4\%), PCV13 (99.4\%), and PPV23 (96.1\%) was observed; however, nine serotypes including 6B $(n=2), 18 F(n=2), 33 A(n=3)$, and $37(n=2)$ were misclassified as possibly vaccine-preventable if assumptions were made based on prevalence. The lack of discrimination of serotypes $6 \mathrm{~A}$ and $6 \mathrm{~B}$ would not be problematic for PCV13, since this vaccine includes coverage for both serotypes; however, serotype $6 \mathrm{~A}$ is not covered by PCV7 or PPV23. Investigations are currently underway to identify novel targets for detection and differentiate of serotypes found in all current pneumococcal vaccines.

While other studies have used oligonucleotide permutations to account for geographical differences in S. pneumoniae serotype distribution (USA, Latin America, Africa, and Asia), ${ }^{[17,24,40]}$ this study showed that oligonucleotides permutations did not affect the performance characteristics of PCR-based serotyping. This study does not preclude additional validation if different permutations are used, or use of ongoing quality assurance controls. Both cmPCR and cmPCRmod suffer from the same limitations where further optimization would be required for accurate discrimination of certain serotypes. On the other hand, cmPCRmod was more cost-effective than cmPCR and reduced the number of PCR reactions were required to identify vaccine-preventable serotypes of $S$. pneumoniae. Overall, serotyping of $S$. pneumoniae isolates using the cmPCRmod reactions could provide significant cost savings for large epidemiological studies such as the active CAP and IPD surveillance conducted by the CIRN SOS Network.

\section{Conflicts of InTEREST Disclosure}

Authors declare that they have no competing interests.

\section{REFERENCES}

[1] Chalmers JD, Campling J, Dicker A, et al. A systematic review of the burden of vaccine preventable pneumococcal disease in UK adults. BMC Pulm Med. 2016; 16: 77. PMid:27169895 http: //dx.doi.org/10.1186/s12890-016-0242-0

[2] McNeil SA, Qizilbash N, Ye J, et al. A retrospective study of the clinical burden of hospitalized all-cause and pneumococcal pneumonia in Canada. Can Respir J. 2015: 17154. http://dx.doi.org/10.

\section{$1155 / 2016 / 3605834$}

[3] O'Brien KL, Wolfson LJ, Watt JP, et al. Hib and Pneumococcal Global Burden of Disease Study Team. Burden of disease caused by Streptococcus pneumoniae in children younger than 5 years: global estimates. Lancet. 2009; 374: 893-902. http://dx.doi.org/10. 1016/S0140-6736(09)61204-6

[4] Said MA, Johnson HL, Nonyane BA, et al. Estimating the burden of pneumococcal pneumonia among adults: a systematic review and 
meta-analysis of diagnostic techniques. PLoS One. 2013; 8: e60273. PMid:23565216 http://dx.doi.org/10.1371/journal. pone. 0060273

[5] Drijkoningen JJ, Rohde GG. Pneumococcal infection in adults: burden of disease. Clin. Microbiol. Infect. 2014; 20: 45-51. PMid:24313448 http://dx.doi.org/10.1111/1469-0691.12 461

[6] World Health Organization (WHO). Estimated Hib and pneumococcal deaths for children under 5 years of age, 2000. 2014. Available from: http://www . who. int/immunization/monitoring_ surveillance/burden/estimates/Pneumo_hib_2000/en/

[7] Bettinger JA, Scheifele DW, Kellner JD, et al. Canadian Immunization Monitoring Program, Active (IMPACT). The effect of routine vaccination on invasive pneumococcal infections in Canadian children, Immunization Monitoring Program, Active 2000-2007. Vaccine. 2010; 28: 2130-2136. PMid:20044050 http://dx.doi.org/10. 1016/j . vaccine. 2009.12.026

[8] Centers for Disease Control and Prevention (CDC). Invasive pneumococcal disease in young children before licensure of 13-valent pneumococcal conjugate vaccine-United States, 2007. MMWR Morb Mortal Wkly Rep. 2010; 59: 253-7. PMid:20224541

[9] Kellner JD, Vanderkooi OG, MacDonald J, et al. Changing epidemiology of invasive pneumococcal disease in Canada, 19982007: update from the Calgary-area Streptococcus pneumoniae research (CASPER) study. Clin. Infect. Dis. 2009; 49: 205-212. PMid:19508165 http://dx.doi.org/10.1086/599827

[10] Demczuk WH, Martin I, Griffith A, et al. Serotype distribution of invasive Streptococcus pneumoniae in Canada after the introduction of the 13-valent pneumococcal conjugate vaccine, 20102012. Can. J. Microbiol. 2013; 59: 778-788. PMid:24313450 http: //dx.doi.org/10.1139/cjm-2013-0614

[11] Bonten MJ, Huijts SM, Bolkenbaas M, et al. Polysaccharide conjugate vaccine against pneumococcal pneumonia in adults. N Engl J Med. 2015; 372: 1114-1125. PMid:25785969 http://dx.doi.o rg/10.1056/NEJMoa1408544

[12] Tomczyk S, Bennett NM, Stoecker C, et al. Use of PCV-13 and PPSV-23 vaccine among adults aged 65 and older: recommendations of the ACIP. MMWR Morb Mortal Wkly Rep. 2014; 63: 822-825. PMid:25233284

[13] National Advisory Committee on Immunization (NACI). Update on the use of 13-valent pneumococcal conjugate vaccine (PNEU-C13 ) in addition to 23 -valent pneumococcal polysaccharide vaccine (PNEU-P-23) in immunocompetent adults 65 years of age and older - Interim Recommendation. 2016.

[14] Public Health Agency of Canada (PHAC). National Advisory Committee on Immunization (NACI). 2015. Available from: http: //www.phac-aspc.gc.ca/naci-ccni/index-eng.php

[15] Austrian R. The Quellung reaction, a neglected microbiologic technique. Mt. Sinai J. Med. 1976; 43: 699-709. PMid:13297

[16] Azzari C, Moriondo M, Indolfi G, et al. Molecular detection methods and serotyping performed directly on clinical samples improve diagnostic sensitivity and reveal increased incidence of invasive disease by Streptococcus pneumoniae in Italian children. J. Med. Microbiol. 2008; 57: 1205-1212. PMid:18809546 http://dx.doi.org/10. 1099/jmm.0.2008/000935-0

[17] Njanpop Lafourcade BM, Sanou O, van der Linden M, et al. Serotyping pneumococcal meningitis cases in the African meningitis belt by use of multiplex PCR with cerebrospinal fluid. J. Clin. Microbiol. 2010; 48: 612-614. PMid:20007384 http://dx.doi.org/10.11 28/JCM. 01402-09

[18] Collard JM, Alio Sanda AK, Jusot JF. Determination of pneumococcal serotypes in meningitis cases in Niger, 2003-2011. PLoS One.
2013; 8: e60432. PMid:23555971 http://dx.doi.org/10.1371 /journal.pone. 0060432

[19] Lang ALS, McNeil SA, Hatchette TF, et al. Detection and prediction of Streptococcus pneumoniae serotypes directly from nasopharyngeal swabs using PCR. J Med Micobiol. 2015; 64: 836-44 PMid:26066632 http://dx.doi.org/10.1099/jmm.0.000097

[20] Jourdain S, Drèze PA, Vandeven J, et al. Sequential multiplex PCR assay for determining capsular serotypes of colonizing S pneumoniae. BMC Infect. Dis. 2011; 11: 100. PMid:21507244 http://dx.doi .org/10.1186/1471-2334-11-100

[21] Dias CA, Teixeira LM, Carvalho MDG, et al. Sequential multiplex PCR for determining capsular serotypes of pneumococci recovered from Brazilian children. J. Med. Microbiol. 2007; 56: 11851188. PMid:17761481 http://dx.doi.org/10.1099/jmm.0.4 7347-0

[22] Miernyk K, Debyle C, Harker-Jones M, et al. Serotyping of Streptococcus pneumoniae isolates from nasopharyngeal samples: use of an algorithm combining microbiologic, serologic, and sequential multiplex PCR techniques. J Clin Microbiol. 2011; 49: 3209-3214. PMid:21775540 http://dx.doi .org/10.1128/JCM . 00610-11

[23] Morais L, Carvalho Mda G, Roca A, et al. Sequential multiplex PCR for identifying pneumococcal capsular serotypes from SouthSaharan African clinical isolates. J. Med. Microbiol. 2007; 56: 11811184. PMid:17761480 http://dx.doi.org/10.1099/jmm.0.4 7346-0

[24] Carvalho MDG, Pimenta FC, Jackson D, et al. Revisiting pneumococcal carriage by use of broth enrichment and PCR techniques for enhanced detection of carriage and serotypes. J. Clin. Microbiol. 2010; 48: 1611-1168. PMid:20220175 http://dx.doi.org/10. 1128/JCM. 02243-09

[25] Pai R, Gertz RE, Beall B. Sequential multiplex PCR approach for determining capsular serotypes of Streptococcus pneumoniae isolates. J. Clin. Microbiol. 2006; 44: 124-131. PMid:16390959 http://dx.doi.org/10.1128/JCM.44.1.124-131.2006

[26] Pimenta FC, Roundtree A, Soysal A, et al. Sequential triplex realtime PCR assay for detecting 21 pneumococcal capsular serotypes that account for a high global disease burden. J. Clin. Microbiol. 2013; 51: 647-652. PMid:23224094 http://dx.doi.org/10.11 28/JCM. 02927-12

[27] Resti M, Moriondo M, Cortimiglia M, et al. Community-acquired bacteremic pneumococcal pneumonia in children: diagnosis and serotyping by real-time polymerase chain reaction using blood samples. Clin Infect Dis. 2010; 51: 1042-1049. PMid:20883110 http://dx.doi.org/10.1086/656579

[28] Richter SS, Heilmann KP, Dohrn CL, et al. Evaluation of pneumococcal serotyping by multiplex PCR and quellung reactions. J. Clin. Microbiol. 2013; 51: 4193-4195. PMid:24025905 http: //dx.doi.org/10.1128/JCM.01876-13

[29] McNeil SA, Shinde V, Andrew MK, et al. Public Health Agency of Canada/Canadian Institutes of Health Research Influenza Research Network (PCIRN) Serious Outcomes Surveillance Network; Toronto Invasive Bacterial Diseases Network (TIBDN). Interim estimates of 2013/14 influenza clinical severity and vaccine effectiveness in the prevention of laboratory-confirmed influenza-related hospitalisation, Canada, February 2014. Euro. Surveill. 2014; 19: 20729. PMid:24626207 http://dx.doi.org/10.2807/1560-7917.ES 2014.19.9.20729

[30] Lovgren M, Spika JS, Talbot JA. Invasive Streptococcus pneumoniae infections: serotype distribution and antimicrobial resistance in Canada, 1992-1995. CMAJ. 1998; 158: 327-331. PMid:9484256

[31] Spellerberg B, Brandt C. Streptococcus. In Murray, P. R., Baron, E. L., Jorgensen, J. H., Landry M. L., \& Pfaller, M. A. (Eds.) Man- 
ual of Clinical Microbiology, American Society of Microbiology, Washington, USA. 2007: 412-429.

[32] Carvalho MDG, Pimenta FC, Gertz RE, et al. PCR-based quantitation and clonal diversity of the current prevalent invasive serogroup 6 pneumococcal serotype, 6C, in the United States in 1999 and 2006 to 2007. J. Clin. Microbiol. 2009; 47: 554-559. PMid:19116353

[33] Pimenta FC, Gertz RE Jr, Roundtree A, et al. Rarely occurring 19Alike cps locus from a serotype $19 \mathrm{~F}$ pneumococcal isolate indicates continued need of serology-based quality control for PCR-based serotype determinations. J Clin Microbiol. 2009; 4:2353-4. PMid: 19439547. http://dx.doi.org/10.1128/JCM.00704-09

[34] Bentley SD, Aanensen DM, Mavroidi A, et al. Genetic analysis of the capsular biosynthetic locus from all 90 pneumococcal serotypes. PLoS Genet. 2006; 2: e31. http://dx.doi .org/10.1371/journ al.pgen. 0020031

[35] Kong F, Brown M, Sabananthan A, et al. Multiplex PCR-based reverse line blot hybridization assay to identify 23 Streptococcus pneumoniae polysaccharide vaccine serotypes. J Clin Microbiol. 2006; 44: 1887-1891. PMid:16672432 http://dx.doi.org/10.1128/JCM $.44 .5 .1887-1891.2006$
[36] Leung MH, Bryson K, Freystatter K, et al. Sequetyping: serotyping Streptococcus pneumoniae by a single PCR sequencing strategy. J Clin Microbiol. 2012; 50: 2419-27. PMid:22553238 http: //dx.doi.org/10.1128/JCM.06384-11

[37] Liyanapathirana V, Ang I, Tsang D, et al. Application of a target enrichment-based next-generation sequencing protocol for identification and sequence-based prediction of pneumococcal serotypes. BMC Microbiol. 2014; 14: 60. PMid:24612771 http://dx.doi.o $\mathrm{rg} / 10.1186 / 1471-2180-14-60$

[38] Mavroidi A, Aanensen DM, Godoy D, et al. Genetic relatedness of the Streptococcus pneumoniae capsular biosynthetic loci. J Bacteriol. 2007; 189: 7841-7855. PMid:17766424 http://dx.doi.org/10. 1128/JB. 00836-07

[39] Tomita Y, Okamoto A, Yamada K, et al. A new microarray system to detect Streptococcus pneumoniae serotypes. J Biomed Biotechnol. 2011; 2011: 352736 .

[40] Centers for Disease Control and Prevention (CDC). PCR deduction of pneumococcal serotypes. http://www. cdc.gov/streplab/p cr.html. (last accessed October 13, 2016). 